\title{
Introducción suave a ideas fundamentales para resolver problemas de programación lineal en enteros por medio de matemáticas recreativas
}

\author{
M.A. Murray-Lasso \\ Unidad de Enseñanza Auxiliada por Computadora \\ D epartamento de Ingeniería de Sistemas. División de Estudios de Posgrado \\ Facultad de Ingeniería, UNAM \\ E-mail: mamurray@servidor.unam.mx
}

(recibido: noviembre de 2003; aceptado: marzo de 2004)

\begin{abstract}
Resumen
Los algoritmos de corte de Gomory para resolver programas lineales en enteros tienen que encontrar una solución entera a un programa lineal obtenido del orig inal al que se le hicieron unos "cortes." La presentacion en los textos de dichos algoritmos, generalmente son muy abstractas y difíciles de seguir, máxime que pocos textos presentan ejemplos en todo detalle donde se vea exactamente qué hace cada corte. En este artículo, se muestran varios ejemplos con soluciones detalladas y con una complejidad creciente de problemas, cuyas soluciones deben ser enteras y positivas utilizando matemáticas recreativas (acertijos matemáticos). Los problemas se resuelven mostrando la utilidad de algunas ideas sencillas para obligar a las soluciones a ser enteras. Como esta idea es nueva y funda mental acerca de los algoritmos de Gomory, ya que las demás son las del algoritmo simplex, el artículo sirve para entender mejor los algoritmos de cortes evitando el misterio que genera la excesiva abstracción y la compleja notación de los textos en la materia.
\end{abstract}

Descriptores: Ecuaciones diofantinas, programación lineal entera, algoritmos de corte, matemáticas recreativas, Gomory.

\footnotetext{
Abstract

Thecut tingal go rithms of $G$ omory for solving lin ear in teger programs find an in teger sol $u$ tion to a linear pro gram ob tained from theorig i nal prob lem to which some "cuts" have been added. Thepres en ta tions given in thetext books that in troducetheseal go rithms aregen er ally ab stract and dif fi cult to visual ise, of ten becausethetexts do not pro vide detailed ex am ples in which the reader can seeclearly what each cut does. In this ar ti cleweuse recreational math emat ics (math puz zles) and giveseleral ex am ples of in creas ing com plex ity to gether with their detailed so lu tions for prob lems in which pos tivein teger so lu tions are required, as means to ex plain ing what is go ing on with the cuts. Theex am ple prob lems are sol ved by show ing the useful ness of some sim pleideas that forcethe so lu tions to bein tegers. Since this is the fun da men tal new idea of Gomory's cut ting al go rithms, given that the other ideas are those already in use by thesim plex al go rithm, thear ti cleshould be useful to help stu dents un derstand better thecutting al go rithms by elim inat ing themystery gen er ated by theex cessiveabstraction and the complex no ta tion of the cor respond ing text books.
}

Keywords: Diophantineequa tions, in teger lin ear program ming, cut ting al go rithms, recreational mathematics, Gomory. 


\section{Introducción}

Una de las principales tareas de la impartición de la ingeniería es enseñar al estudiante a plantear y re solver problemas. Dada la enorme variedad de posibles clases de problemas que se le pueden presentar al fu turo ingeniero y dado que no se ha encontrado la manera de englobarlos todos en un marco y teoría general, no parece haber más remedio que tratar de coleccionar buenos problemas paradigmáticos que sirvan de modelo a las principales familias de problemas típicos que se presentan en la práctica. En la solución de dichos problemas aparecen conceptos y métodos de aplicabilidad más o menos general que conviene que los estudiantes incorporen a su herramental profesional. Algunos de ellos son: el establecimiento de ecuaciones matemáticas para describir las condiciones que se le imponen a las cantidades que intervienen en un problema; el análisis de un elemento típico variable (por ejemplo, un elemento de volumen) para el cual se escriben ecuaciones correspondientes a leyes de conservación y otras que al hacer ten der las dimensiones a cero dan lugar a ecuaciones diferenciales, integrales o integro-diferenciales que debe satisfacer todo el fenómeno; el método de aproximaciones sucesivas para re solver una o varias ecuaciones, ya sean algebraicas o diferenciales; el uso de simulación en la computadora para explorar el espacio de posibilidades y contestar preguntas: ¿qué tal si...?; el uso de técnicas de "trepar colinas" (hill climbing) para determinar parámetros en diseños; la simplificación de modelos ignorando algunas de las condiciones, resolviendo y volviendo a imponer las condiciones ignoradas; etc.

Por la limitación de tiempo en las carreras de ingeniería, en muchos casos no es posible re solver en clase problemas realistas en detalle, pues el sim ple planteamiento del problema y el acopio de datos llevaría mucho más tiempo que el disponible. Debido a ello, es necesario en muchos casos re solver problemas "de juguete," altamente simplificados para dar a los estudiantes "una probadita" de lo que se trata. Así, se resuelven circuitos eléctricos con 5 o 6 elementos; armaduras con 7 a 9 barras; redes de transporte con 2 orígenes y 3 destinos; planes de producción con 3 productos; etc.

La computadora ha venido a enriquecer la educación de los estudiantes en forma impresionante, pues cuando se cuenta con el software y equipo adecuados se pueden realizar proyectos realistas; sin em bargo, al estar formando profesionales, no basta con enseñarles cómo operar un programa en forma de receta; es necesario que los estudiantes comprendan los principios, conceptos y procesos detrás de los programas y que adquieran el criterio suficiente para utilizarlos crítica y creativamente. Para lograr esto, es necesario que los estudiantes de verdad sepan re solver problemas.

En este artículo se presenta una introducción muy ligera e informal de algunas ideas fundamentales que son utilizadas para resolver problemas de programación lin eal en enteros usando como paradigma una fa milia de acertijos populares en la literatura de las matemáticas recreativas. Se presenta en detalle una técnica muy simple, que sin embargo, no es ampliamente conocida entre estudiantes de ingeniería ni de otras disciplinas como economía, administración, física y ni siquiera matemáticas, excepto los especialistas en teoría de números. La intención de más largo alcance es proponer que los profesores de ingeniería coleccionen problemas similares junto con los conceptos y métodos de solución asociados para integrar una "caja de herramientas" para los estudiantes de ingeniería que les será de utilidad en su vida profesional.

Al estudiar algoritmos como los de Gomory para re solver programas lineales enteros, en gen eral los estudiantes ya estudiaron el al- goritmo simplex, y por lo tanto, conocen los trucos utilizados para convertir desigualdades en igualdades, ir cambiando de base sin que las variables se vuelvan negativas e ir aumentando la función objetivo con cada iteración. La verdadera novedad en programación en enteros es obligar a las soluciones a que sean enteras y no fraccionarias. 
Es en esta circunstancia en la que se concentra este artículo. Una vez que el estudiante do mina la idea fundamental, le será mucho más fácil entender los algoritmos de cortes de Gomory. (Hu, 1969); (Dantzig, 1963).

\section{Los acertijos como fuente de materiales para enseñar la resolución de problemas}

No cabe duda que una de las características de la especie humana es su gusto por el juego. La popularidad de los deportes competitivos, juegos de azar, juegos electrónicos, juegos de salón, magia, juguetes para niños y adultos, acertijos, crucigramas, y otros pasatiempos lo demuestra.

La pasión con la que los niños juegan Nintendo y la cantidad de tiempo que están dispuestos a dedicarle nos indican el bien que haríamos en incorporarlos al proceso de enseñanza-aprendizaje. Buenas fuentes de temas para practicar el arte de resolver problemas son los acertijos, particularmente los acertijos matemáticos. Existe una rica literatura, tanto en libros como en revistas populares sobre el tema (Dudeney, 1967), (Gardner, 1961) y (Perelman, 1983). Casi todos los libros proporcionan respuestas, pero adolecen de la falta de explicación detallada sobre los métodos organizados y confiables de solución, así como los conceptos y procesos asociados que pueden servir para re solver problemas similares.

Una razón válida de su ausencia es que ocuparían una gran cantidad de espacio. La propuesta es seleccionar aquellos problemas de los cuales más se puede aprender y proporcionar métodos de solución en detalle para así convertir los libros de acertijos matemáticos en materiales educativos útiles en el aprendizaje del arte de resolución de problemas.

\section{La familia de problemas lineales diofantinos}

Diofantes, un matemático que vivió en Alejandría alrededor del año 250 de nuestra era, del cual se sabe muy poco por referencias indirectas, es probablemente el algebrista más distinguido de la Grecia de su época. Su libro Aritmética que en realidad es un libro rudimentario de álgebra, nos ha llegado incompleto a nuestros días. En su honor, las ecuaciones para las cuales se buscan soluciones racionales y en enteros se llaman ecuaciones diofantinas (Ore, 1988), (Rouse, 1960) y (Struik, 1967). De ellas las lineales son las más fáciles de resolver. Una ecuación lineal con coeficientes fraccionarios con más de una vari able para la cual se busca una solución racional se resuelve trivialmente dándole valores racionales arbitrarios a todas las variables menos una y despejando la vari able res tante en términos de las demás. Más interesantes son las ecuaciones lineales con coeficientes enteros con más de una vari able, para la cual se buscan soluciones enteras, en muchas ocasiones no negativas. Frecuentemente existe más de una solución entera, no negativa, y para forzar una solución única se imponen condiciones adicionales, frecuentemente encontrar la solución más pequeña. Este problema es un caso muy sim ple de los que en investigación de operaciones se conocen como un problema de programación lineal en enteros. El problema descrito ya es lo suficientemente complicado para que se le haya utilizado como acertijo desde tiempos remotos, antes de la invención del álgebra y menos todavía de la investigación de operaciones.

Podríamos tratar el problema utilizando una notación abstracta y un vocabulario especializado; sin em bargo, el espíritu del artículo es tratarlo de manera in for mal, por lo que se presenta por medio de varios acertijos de complejidad creciente (Ore, 1988).

1. En un manuscrito del siglo $X$ (se cree que es copia de una colección de acertijos preparados para Carlomagno) aparece el siguiente problema: Cuando se distribuyen entre 100 per so nas 100 costales de granos en forma tal que cada hombre recibe 3 costales, cada mujer recibe 2 y 
cada niño recibe $1 / 2$ costal, ¿Cuántos hombres, mujeres y niños hay?

2. Tomado del Álgebra de Euler:

Escribir el número 25 como la suma de dos enteros positivos, uno di vis i ble entre 2 y otro divisible entre 3.

3. Tomado del Álgebra de Euler:

Un hombre compra caballos y vacas pagando un total de \$1 770. Cada caballo cuesta \$31 y cada vaca \$21 ¿Cuántos caballos y cuántas vacas compró?

4. En la revista Saturday Evening Post del 9 de octubre de 1926, apareció una leve variante del siguiente problema:

Cinco hom bres y un mono naufragaron en una isla desierta y se pasaron el primer día juntando cocos para comer. Los pusieron en un gran montón y se fueron a dormir. Cuando todos estaban dormidos, uno de los hom bres despertó y pensando que podría haber pleito en la repartición de los cocos a la mañana siguiente, decidió tomar su parte. Dividió los cocos en 5 montones iguales y le sobró un coco que se lo dio al mono. Enterró uno de los montones, juntó los demás montones en un gran montón y se fue a dormir. Cada uno de los demás hom bres se fueron despertando a horas diferentes e hicieron lo mismo, cada uno encontrando que al dividir los cocos en 5 partes iguales sobraba un coco que se lo dieron al mono y cada uno enterrando la quinta parte de los cocos (excepción hecha del coco del mono) y juntando los restantes en un gran montón. En la mañana siguiente, los hombres divi- dieron los cocos restantes en 5 par tes iguales y sobró un coco que se lo dieron al mono. Seguramente todos los hombres se dieron cuenta en la mañana que faltaban cocos; sin embargo, sabiendo que ellos habían hecho trampa no dijeron nada. ¿Cuántos cocos había orig i nal- mente? (Gardner, 1961).

Este problema causó tanto interés que la semana siguiente a la fecha en que apareció, la revista recibió 2,000 cartas y el autor continuó recibiendo cartas durante 20 años pidiendo la respuesta 0 proponiendo soluciones.

\section{La formulación de problemas diofantinos lineales}

Los problemas exhibidos en la sección anteriorse podrían resolver por ensayo y error, sin plantear ecuaciones y sin utilizar técnicas algebraicas formales. Los primeros dos, particularmente el segundo, los puede re solver fácilmente el hom bre de la calle. El tercero ya resulta laborioso para encontrar una solución y el cuarto resulta francamente muy laborioso resolverlo sin álgebra debido a la presencia de números grandes. En este artículo exhibiremos una técnica segura y eficaz para re solver este tipo de problemas que aparecen con frecuencia en la literatura de acertijos matemáticos (Ore, 1988) y (Perelman, 1983). La técnica siempre lleva a una solución cuando existe y detecta cuando no hay solución.

El primer paso para resolver los acertijos mostrados es plantear una ecuación matemática, lo cual hacemos para cada uno de los problemas:

1. Si llamamos con las letras $h, m, y n$ al número (todavía desconocido) de hombres, mujeres y niños, respectivamente, en vista de que el total de personas es 100 podemos escribir la ecuación

$$
h+m+n=100
$$

Por otra parte, el número de costales que recibieron los hom bres es $3 \mathrm{~h}$, el que recibieron las mujeres es $2 \mathrm{~m}$ y el que recibieron los niños es $1 / 2 n$, y como el total de costales es 100 se puede escribir la ecuación

$$
3 h+2 m+1 / 2 n=100
$$

Tenemos dos ecuaciones con 3 incógnitas. Las incógnitas deben ser números enteros no negativos. Ya podríamos concluir de las ecuaciones y 
las características de las incógnitas que ninguna de las incógnitas debe exceder 100 y que el número de niños debe ser par (para que no aparezcan fracciones).

2. Para que uno de los sumandos sea divisible entre 3 y el otro entre 2 , podemos escribirlos $3 x$ y $2 y$, donde $x$ e y son números enteros. Los dos sumandos suman 25 , entonces:

$$
3 x+2 y=25
$$

En este caso tenemos una ecuación con dos incógnitas $x$ e y enteras y no negativas.

3. Si representamos con c el número de caballos y con v el número de vacas, la cantidad a pagar será $31 c+21 v$ la cual debe ser igual al total pagado, 1 770. Por lo tanto, la ecuación es:

$$
31 c+21 v=1770
$$

4. Si llamamos $\mathrm{N}_{0}$ al número original de cocos, $\mathrm{N}_{1}$ los que quedaron en el montón general después de que el primer hom bre enterró su parte, $\mathrm{N}_{2}$ los que quedaron en el montón general después de que el segundo hombre enterró su parte, $N_{3}, N_{4}, N_{5}$ los que quedaron en el montón gen eraldespués de que el tercer, cuarto y quinto hom bre enterraron su parte, respectivamente, y $F$ a la cantidad que le tocó a cada hombre en la repartición de la mañana, se tiene

$$
\begin{aligned}
N_{1} & =4\left(N_{0}-1\right) / 5 \\
N_{2} & =4\left(N_{1}-1\right) / 5 \\
N_{3} & =4\left(N_{2}-1\right) / 5 \\
N_{4} & =4\left(N_{3}-1\right) / 5 \\
N_{5} & =4\left(N_{4}-1\right) / 5 \\
\mathrm{~F} & =\left(N_{5}-1\right) / 5
\end{aligned}
$$

Las ecuaciones anteriores se pueden escribir

$$
\left.\begin{array}{l}
4 N_{0}=5 N_{1}+4 \\
4 N_{1}=5 N_{2}+4 \\
4 N_{2}=5 N_{3}+4 \\
4 N_{3}=5 N_{4}+4 \\
4 N_{4}=5 N_{5}+4 \\
N_{5}=5 F+1
\end{array}\right]
$$

Tenemos 6 ecuaciones con 7 incógnitas ente- ras, no negativas y deseamos encontrar el número más chico NO com pat i ble con las ecuaciones.

\section{Conversión a una ecuación lineal diofantina con 2 variables}

Los cuatro problemas planteados se pueden reducir a la solución en enteros no negativos de una ecuación con 2 incógnitas como se muestra a continuación:

1. En este problema tenemos 2 ecuaciones con 3 incógnitas. Una de las incógnitas se puede eliminar con una de las ecuaciones. Despejando $\mathrm{h}$ de la primera ecuación y reemplazando su valor en la segunda se tiene

$$
\begin{aligned}
& h=100-m-n \\
& 3(100-m-n)+2 m+1 / 2 n=100, \text { simp lificand } 0 \\
& 2 m+5 n=400
\end{aligned}
$$

2. Ya se tiene la ecuación en la forma deseada.

3. Ya se tiene la ecuación en la forma deseada.

4. Se pueden eliminar las incógnitas $N_{1}$, $N_{2}, N_{3}, N_{4}, N_{5}$ usando las últimas 5 ecuaciones para que la primera ecuación sólo tenga $N_{0}$ y $F$ como variables.

a) Multiplíquese la última ecuación del grupo (1) por 5 y reemplace el $\mathrm{N}_{5}$ de la penúltima ecuación con su valor para obtener

$$
4 N_{4}=25 F+9
$$


Con esto queda eliminada $N_{5}$.

b) Multiplique por 5 la ecuación (5') y por 4 la cuarta ecuación del grupo (1) y elimínese $N_{4}$ en esta última por sustitución obteniendo

$$
16 N_{3}=125 F+61
$$

c) Multiplique por 16 la tercera ecuación de grupo (1) y por 5 la ecuación (4') y elimine $N_{3}$ por sustitución obteniendo

$$
64 N_{2}=625 F+369
$$

d) Multiplique por 64 la segunda ecuación del grupo (1) y por 5 la ecuación (3') y elimínese $N_{2}$ por sustitución obteniendo

$$
256 N_{1}=3125 F+2101
$$

e) Multiplique por 256 la primera ecuación del grupo (1) y por 5 la ecuación (2') obteniendo

$$
1024 N_{0}=15625 F+11529
$$

Nótese que para no introducir fracciones se evitó en todo momento la operación de división en el proceso de eliminación. (Alternativamente se podría haber introducido la división y manejado fracciones para el final, calculando el mínimo común di vi sor de todas las fracciones, multiplicar por dicha cantidad todas las fracciones para convertirlas en enteros.

\section{Método simple para la solución de una ecuación lineal diofantina con dos variables}

Aunque existen métodos que utilizan fracciones continuadas para resolver ecuaciones lineales diofantinas, presentamos un método conceptualmente más sim ple para resolverlas. Para facilitar la comprensión del método, resolveremos las ecuaciones correspondientes a los 4 acertijos presentados en vez de hablar en abstracto e introducir una notación complicada. Comenzamos con el acertijo 2 que es el caso más sencillo.

En la ecuación $3 x+2 y=25$ comenzamos por despejar la variable y que es la que tiene coeficiente con menor valor absoluto,

$$
y=(25-3 x) / 2=12-x+(1-x) / 2
$$

Hemos separado la parte entera de la expresión en $\mathrm{x}$ de la parte fraccionaria. Como $\mathrm{x}$ debe ser entera, el último término también debe ser entero. Llamemos a dicho entero $x_{1}$, el cual debe satisfacer

$$
x=1-2 x_{1}
$$

Deseamos expresar y en términos de $x_{1}$, por lo que reemplazamos el valor de $x$ en (7) por el valor dado por (8) y obtenemos

$$
y=12-\left(1-2 x_{1}\right)+x_{1}=11+3 x_{1}
$$

La solución está dada por la ecuación (8) y la última ecuación que expresan $x$ e y en términos del parámetro entero $x_{1}$. Si no se obliga a $x$ e y a ser positivas, la solución obtenida genera una infinidad de pares $x$, y que satisfacen la ecuación inicial. Por ejemplo, si tomamos $x_{1}=1$, se obtiene $y=14, x=$ -1 y los dos sumando serán $3(-1)=-3$ y $2(14)=28$. Se cumple que $-3+28=25$. Con $x_{1}=2,3,4, \ldots$ se obtiene una infinidad de soluciones diferentes en que uno de los sumandos es nega- tivo. Si queremos obligar a los sumandos a ser positivos, imponemos

$$
y=11+3 x_{1}>0, x=1-2 x_{1}>0
$$

de donde $x_{1}>-11 / 3$ y $x_{1}<1 / 2,0$ sea, que los posibles valores de $x_{1}$ para que tanto $x$ como $y$ sean positivos son $0,-1,-2,-3$, los cuales dan los siguientes sumandos $s_{1}$ y $s_{2}$ 


$\begin{array}{ccccc}x_{1} & x & y & s_{1}=2 y & s_{2}=2 y \\ 0 & 1 & 11 & 3 & 22 \\ -1 & 3 & 8 & 9 & 16 \\ -2 & 5 & 5 & 15 & 10 \\ -3 & 7 & 2 & 21 & 4\end{array}$

Para el primer acertijo cuya ecuación en dos vari ables es $2 m+5 n=400$, se despeja $m$, que es la vari able con coeficiente de menor valor absoluto, obteniendo

$$
m=(400-5 n) / 2=200-2 n-n / 2
$$

Llamamos $x_{1}=n / 2$ o lo que es lo mismo $n=2 x_{1}$. Eliminando $n$ se tiene

$$
m=200-4 x_{1}-x_{1}=200-5 x_{1}
$$

donde $x_{1}$ es cualquier entero. Para terminar con hom bres, mujeres o niños no negativos imponemos

$$
m=200-5 x_{1} \geq 0, n=2 x_{1} \geq 0, h=100-m-n \geq 0
$$

de donde $x_{1} \geq 0, x_{1} \leq 40, x_{1} \geq 331 / 3$. Los posibles valores de $x_{1}$ son: $34,35,36,37,38,39,40$.

Las soluciones están dadas por:

\begin{tabular}{ccccccccc}
\hline $\mathbf{x} \mathbf{1}$ & $\mathbf{h}$ & $\mathbf{m}$ & $\mathbf{n}$ & $\mathbf{3 h}$ & $\mathbf{2 m}$ & $\mathbf{n} / \mathbf{2}$ & $\mathbf{h +} \mathbf{m}+\mathbf{n}$ & $\mathbf{3} \mathbf{h + 2} \mathbf{n + ( 1 / 2 ) \mathbf { n }}$ \\
\hline 34 & 2 & 30 & 68 & 6 & 60 & 34 & 100 & 100 \\
35 & 5 & 25 & 70 & 15 & 50 & 35 & 100 & 100 \\
36 & 8 & 20 & 72 & 24 & 40 & 36 & 100 & 100 \\
37 & 11 & 15 & 74 & 33 & 30 & 37 & 100 & 100 \\
38 & 14 & 10 & 76 & 42 & 20 & 38 & 100 & 100 \\
39 & 17 & 5 & 78 & 51 & 10 & 39 & 100 & 100 \\
40 & 20 & 0 & 80 & 60 & 0 & 40 & 100 & 100 \\
\hline
\end{tabular}

Para reducir el número de soluciones se podrían agregar condiciones al problema. Por ejemplo, que el número de hombres sea divisible entre 7 (daría una solución única.) Que el número de mujeres sea primo (también da solución única.) Que haya menos mujeres que hom bres (reduciría el número de soluciones a tres.)

Para re solver el tercer acertijo, cuya ecuación es $31 \mathrm{c}+21 \mathrm{v}=1770$ comenzamos despejando $\mathrm{v}$

$$
V=(1770-31 c) / 21=84-c+(6-10 c) / 21
$$

Llamamos $x_{1}$ al último término, o lo que es lo mismo $21 x_{1}+10 c=6$. Esta ecuación es sim i lar a la orig i nal pero con coeficientes de menor valor absoluto (siempre es así). Le aplicamos a la nueva ecuación la misma técnica. Despejamos la variable con coeficiente de menor valor absoluto obteniendo

$$
c=\left(6-21 x_{1}\right) / 10=-2 x_{1}+\left(6-x_{1}\right) / 10
$$

Llamamos $x_{2}$ al último término, lo que equivale a escribir 


$$
x_{1}=6-10 x_{2}
$$

Notamos que ya podemos expresar todas las variables en términos de $x_{2}$ sin meter fracciones, por lo que eliminamos las $x_{1}$ y escribimos

$$
\begin{aligned}
c= & -2\left(6-10 x_{2}\right)+x_{2}=-12+21 x_{2}, v=84 \\
& +12-21 x_{2}+6-10 x_{2}=102-31 x_{2}
\end{aligned}
$$

Para asegurar que $c$ y $\vee$ son positivas imponemos

$c=-12+21 x_{2}>0, v=102-31 x_{2}>0$,

de donde $x_{2}>4 / 7, x_{2}<39 / 31$. Por lo tanto, los valores enteros de $x_{2}$ posibles son $x_{2}=1,2,3$ que generan los valores $c=9,30,51$ y $v=71,40,9$. De las tres soluciones se podría obligar una solución única si se agrega la condición que son más caballos que vacas, en cuyo caso la solución es: Número de Caballos $=51$, Número de Vacas $=9$.

Finalmente, pasamos al acertijo de los hombres, los cocos y el mono. (Este problema ha aparecido varias veces en la literatura matemática Gardner (1961), Moritz (1928), Piele y Wood (1980)). La ecuación inicial es $1024 \mathrm{~N}_{0}=15625 \mathrm{~F}+$ 11529. Despejamos $N_{0}$ que es la variable con coeficiente con menor valor absoluto y obtenemos

$\mathrm{N}_{0}=15 \mathrm{~F}+11+(265 \mathrm{~F}+265) / 1024=15 \mathrm{~F}+$

$$
11+x_{1}
$$

Donde llamamos $x_{1}$ al último término. Se cumple que $1024 x_{1}=265 F+265$. Dado que $F$ tiene el coeficiente con mínimo valor absoluto, lo despejamos y obtenemos

$$
F=-1+3 x_{1}+229 x_{1} / 265=-1+3 x_{1}+x_{2}
$$

Por la definición de $x_{2}$ se cumple que $265 x_{2}=$ $229 x_{1}$, de donde podemos despejar $x_{1}$ y obtener

$$
x_{1}=x_{2}+36 x_{2} / 229=x_{2}+x_{3}
$$

Por la definición de $x_{3}$ se cumple $229 x_{3}=36 x_{2}$. Por ser la vari able con coeficiente de mínimo valor absoluto despejamos $x_{2}$ para obtener

$$
x_{2}=6 x_{3}+13 x_{3} / 36=6 x_{3}+x_{4}
$$

Por definición $x_{4}$ satisface $36 x_{4}=13 x_{3}$. Despejamos $x_{3}$ yobtenemos

$$
x_{3}=2 x_{4}+10 x_{4} / 13=2 x_{4}+x_{5}
$$

donde por definición $x_{5}$ satisface $13 x_{5}=10 x_{4}$. Despejamos $x_{4}$ y obtenemos

$$
x_{4}=x_{5}+3 x_{5} / 10=x_{5}+x_{6}
$$

$x_{6}$ satisface por definición $10 x_{6}=3 x_{5}$. De aquí despejamos $x_{5}$ y obtenemos

$$
x_{5}=3 x_{6}+x_{6} / 3=3 x_{6}+x_{7}
$$

Por definición $x_{7}$ satisface

$$
3 x_{7}=x_{6}
$$

Hemos logrado eliminar todas las fracciones y estamos en condiciones de expresar todas las variables intermedias y las originales en términos de la última variable definida, $x_{7}$. Por (16) la ecuación (15) queda

$$
x_{5}=9 x_{7}+x_{7}=10 x_{7}
$$

por lo que (14) queda

$$
x_{4}=10 x_{7}+3 x_{7}=13 x_{7}
$$

y entonces (13) queda

$$
x_{3}=26 x_{7}+10 x_{7}=36 x_{7}
$$

y (12) entonces queda

$$
x_{2}=216 x_{7}+13 x_{7}=229 x_{7}
$$


lo que nos permite escribir (11) como sigue:

$$
x_{1}=229 x_{7}+36 x_{7}=265 x_{7}
$$

Ahora podemos escribir (10) como sigue:

$$
F=-1+795 x_{7}+229 x_{7}=-1+1024 x_{7}
$$

Y finalmente (9) la podemos expresar

$$
N_{0}=-15+15(1024) x_{7}+11+265 x_{7}=-4+
$$

Tenemos en (17) y (18) las dos incógnitas de la ecuación orig i nal

$$
1024 N_{0}=15625 F+11529
$$

despejadas y expresadas en términos de una variable entera arbitraria. Si nos interesaran todas las soluciones enteras podríamos darle valores arbitrarios enteros $a x_{7}$ y obtener diferentes soluciones para $\mathrm{Fy} \mathrm{N}_{0}$ que satisfarían (19). Por ejemplo, si tomamos $x_{7}=0$ se obtiene $N_{0}=-4, F=-1$ y la ecuación (19) expresa la identidad $-4096=$ -4096. Como en realidad el problema pide solución positiva, imponemos las desigualdades

$$
F=-4+15625 x_{7}>0, N_{0}=-1+1024 x_{7}>0
$$

De donde $x_{7}>4 / 15625 \quad x_{7}>1 / 1024$, es decir, los valores permitidos para $x_{7}$ son: $x_{7}=1,2$, 3 .... Se ve claramente que, dado que los coeficientes de $x_{7}$ en las expresiones para $N_{0}$ y $F$ son positivos, los valores más pequeños para $\mathrm{N}_{0} \mathrm{y}$ $F$ corresponden a $x_{7}=1$. La respuesta final es entonces:

$N_{0}=-1+1024=1023, F=-4+15625=15621$

El número más pequeño de cocos para que pueda suceder lo relatado es 15621. En la mañana siguiente los hom bres se repartieron 1023 cocos.

Aquí se detalla la solución completa del problema:
El primer hombre encontró $N_{0}=15621$ cocos; le dio un coco al mono y le quedaron 15620 cocos los cuales dividió en cinco montones de 15620 / 5 $=3124$ cocos. Enterró esta cantidad de cocos y dejó fuera en un gran montón 4(3124)=12496 cocos. El segundo hom bre le dio un coco al mono y dividió los restantes 12495 cocos en 5 montones iguales de $12494 / 5=2499$ cocos. Enterró uno de los montones y dejó en un gran montón 4(2499)= 9996 cocos. El tercer hombre le dio un coco al mono y dividió los 9995 cocos restantes en 5 montones de 9995/5=1999 cocos cada uno, enterrando uno de los montones y dejando fuera en un gran montón de $4(1999)=7996$ cocos. El cuarto hom bre le dio un coco al mono y dividió los restantes 7995 cocos en 5 montones iguales de $7995 / 5=1599$ cocos cada uno, habiendo enterrado uno de los montones, dejó fuera 4(1599)= 6396 cocos en un gran montón. El quinto hom bre le dio un coco al mono y de los 6395 cocos restantes formó 5 montones de 6395/5=1279 cocos cada uno, enterró uno de los montones y dejó en un gran montón 4(1279)=5116 cocos. En la mañana, al repartir los cocos entre los 5 hom bres, se le dio un coco al mono y de los 5115 cocos restantes le tocó a cada hombre

$F=5115 / 5=1023$ cocos. En la tabla se muestran las cantidades de cocos que cada quien logró subrepticiamente o no:

\begin{tabular}{cccc}
\hline & Enterrados & $\begin{array}{c}\text { Repartidos } \\
\text { en la } \\
\text { mañana }\end{array}$ & Total \\
\hline Hombre 1 & 3124 & 1023 & 4147 \\
Hombre 2 & 2499 & 1023 & 3522 \\
Hombre 3 & 1999 & 1023 & 3022 \\
Hombre 4 & 1599 & 1023 & 2622 \\
Hombre 5 & 1279 & 1023 & 2302 \\
Mono & & & 6 \\
\cline { 3 - 4 } & \multicolumn{2}{c}{ Cocos iniciales } & 15621 \\
\hline
\end{tabular}

\section{Conclusiones}

El problema de los náufragos, el mono y los cocos es un problema de programación lin eal en enteros 
(Hu, 1969) que se podría plantear matemáticamente como sigue:

$$
\begin{aligned}
& \text { Min } N_{0} \\
& \text { Sujeto a: } \\
& 4 N_{0}-5 N_{1}=4 \\
& 4 N_{1}-5 N_{2}=4 \\
& 4 N_{2}-5 N_{3}=4 \\
& 4 N_{3}-5 N_{4}=4 \\
& 4 N_{4}-5 N_{5}=4 \\
& N_{5}-5 F=1 \\
& N_{0}, N_{1}, N_{2}, N_{3}, N_{4}, N_{5}, F \geq 0 \text { y enteros. }
\end{aligned}
$$

Este problema lo resolvimos en el artículo por el siguiente método:

Por medio de transformaciones lineales elementales se usaron todas las ecuaciones menos una para reducir el sistema de ecuaciones a una sola ecuación con 2 incógnitas $N_{0}$ y F). Para asegurar que la solución sería entera se separaron las partes enteras y fraccionarias de la expresión donde quedaba despejada la variable cuyo coeficiente tuviera mínimo valor absoluto. La parte fraccionaria se igualaba con una nueva variable entera y se escribía una ecuación lineal con coeficientes enteros que deberían satisfacer las variables originales y la nueva variable. Esta nueva ecuación tenía coeficientes más pequeños que la ecuación de la cual provenía. Si todas las vari ables se podían expresar en términos de las nuevas variables definidas (variables parámetro) ya se contaba con una respuesta factible entera; en caso contrario, se volvía a aplicar la técnica reduciendo en cada caso el valor absoluto de los coeficientes hasta llegar a la unidad. Para asegurar que los valores de las variables son positivos se imponen desigualdades que limitan los posibles valores permitidos para las vari ables parámetro. Para los casos en que hay sólo una variable parámetro y que hay varios valores posibles, es sencillo determinar cuál valor minimiza la función objetivo examinando los signos de los coeficientes de la variable parámetro.
En realidad, el énfasis del artículo se concentró en dos ideas simples cuya presentación se hizo a través de ejemplos con complejidad creciente: a) eliminar variables con ecuaciones sin introducir fracciones e introducir variables adicionales para las partes fraccionarias de las expresiones, variables cuya definición lleva a ecuaciones lineales con coeficientes, enteros de la misma naturaleza y más pequeños en valor absoluto que el problema original, por lo tanto, atacables recursivamente con la misma técnica. Al ir disminuyendo los valores absolutos de los coeficientes eventualmente aparece la unidad como coeficiente de la última vari able definida, lo cual permite despejarla en términos de vari ables enteras multiplicadas por coeficientes enteros. Esto establece que en un número finito de pasos se puede obtener la solución en términos de vari ables a las cuales se les pueden dar valores arbitrarios enteros y producen soluciones también enteras. Los demás pasos de los algoritmos de corte de Gomory llevan el propósito de optimizar la función objetivo y no permitir que las variables se vuelvan negativos, para lo cual se usan los mismos trucos que el algo ritmo sim plex.

Aunque existe una teoría formal para la solución del tipo de ecuaciones lineales diofantinas como las que se trataron en el artículo, teoría que forma parte de la teoría de congruencias, máximo común divisor, y fracciones continuadas de la teoría de los números (Ore, 1988), (Kinchin, 1964), (Demidovich y Maron, 1976), el autor considera mucho más didáctico resolver los problemas como se describió en el artículo y recomienda que dicha teoría se utilice después de que los alumnos adquieran suficiente práctica con los métodos más simples. Una encuesta personal reveló que las ideas presentadas en este artículo no parecen ser ampliamente conocidas por per so nas ajenas a la Teoría de los Números.

Martin Gardner, famoso autor de libros de acertijos y ex jefe de la sección de juegos matemáticos de la revista ScientificAmerican, dedica un capítulo al problema de los hom bres, el mono y los cocos y le da al lector la impresión de que el 
problema requiere conocimientos complejos y cálculos laboriosos (Gardner, 1961). Hace pasar por las manos de un Premio Nobel en Física, P. A. M. Dirac, y de otros famosos profesores de las mejores universidades de la Gran Bretaña la solución que presenta de este problema, la cual se encuentra por ensayo y error con base en la feliz idea de introducir cocos negativos (recordar que una de nuestras soluciones en números negativos fue -4 cocos iniciales). En este artículo hemos mostrado que la solución del problema se puede lograr con certeza y eficiencia con ideas muy simples y pocos cálculos. El haber planteado varias ecuaciones simultáneas se hizo con el propósito de ilustrar la técnica de eliminación de variables sin introducir fracciones. Es fácil llegar a la ecuación diofantina lin eal con las vari ables $\mathrm{N}_{0}$ y $F$ de un solo golpe pues la ecuación:

$$
F=\frac{1}{5}\left[\frac{4}{5}\left[\frac{4}{5}\left[\frac{4}{5}\left[\frac{4}{5}\left[\frac{4}{5}\left[\mathrm{~N}_{0}-1\right]-1\right]-1\right]-1\right]-1\right]-1\right]
$$

expresa la cantidad repartida la mañana siguiente en términos de la cantidad inicial de cocos. Al eliminar los corchetes y los denominadores se obtiene la ecuación (19) del artículo.

\section{Referencias}

Anning N. (1951) Monkeys and Coconuts. The $M$ athematics Teacher, Vol. 54, No. 8, pp. 560-562.

Bowden J. (1936). The Problem of the Dishonest Men, the Monkeys, and the Coco nuts. Special Topics in Theo ret ical A rith metic, Lancaster Press, Inc., Lancaster, PA, pp. 203-212.

Dantzig G.B. (1963). Linear Programming and Extensions. Princeton University Press, Princeton, NJ, pp. 514-550.

Demidovich B.P. y Maron I.A. (1976). Computational Mathematics. Mir Publishers, Moscú, Capítulo 2: Some Facts from the Theory of Continued Frac tions, pp. 55-76.

Dudeney E. (1967). 536 Puzzles \& Curious Problems.Charles Scribner's Sons, New York, p. 3.
Gardner M. (1961). The $2^{\text {nd }}$ Scientific American Book of Mathematical Puzzles and Diversions. Simon and Schuster, New York, pp. 104-111.

Hu T.C. (1969). Integer Programming and Network Flows. Addi son-Wesley Publishing Company, Reading, MA.

Kinchin A.Y. (1964). Continued Fractions. Phoenix Books, The University of Chicago Press, Chicago.

Kirchner R.B. (1960). The Gener al ized Co conut Problem. TheA merican $M$ ath ematical M onthly, Vol. 67, No. 6, pp. 516-519.

Moritz R.E. (1928). Solu tion to Problem 3,242. The American Mathematical M onthly, Vol. 35, pp. 47-48.

Ore 0. (1988). Number Theory and Its History. Dover Publi ca tions, Inc., N ew York.

Perelman Ya. (1983). Álgebra recreativa. Ediciones Quinto Sol, SA, México, Capítulo 4: LaS ecuaciones de Diofanto, pp. 128-159.

Piele D.T. y Wood L.E. (1980). Thinking Strategies with the Computer, en AhI D.H. y Green B. (editores). The Best of Creative Computing, Vol. 3, Creative Computing Press, Morristown, NJ, pp. 205-224.

Rouse-Ball W.W. (1960). A Short Account of the $H$ istory of $M$ athematics. Dover Publications, Inc., New York, pp. 103-110.

Struik D.J. (1967). A Concise History of Mathematics. Dover Publi ca tions, Inc., New York, pp. 61-62. 
DOI: http://dx.doi.org/10.22201/fi.25940732e.2005.06n1.004

Introducción suave a ideas fundamentales para resolver problemas de programación lineal ...

\section{Semblanza del autor}

M arco A ntonio M urray-Lasso. Realizó la licenciatura en ingeniería mecánica-eléctrica en la Facultad de Ingeniería de la UNAM. EI Instituto de Tecnología de Massachussetts (MIT) le otorgó los grados de maestro en ciencias en ingeniería eléctrica y doctor en ciencias cibernéticas. En México, ha laborado como investigador en el Instituto de Ingeniería y como profesor en la Facultad de Ingeniería (UNAM) durante 43 años; en el extranjero, ha sido asesor de la NASA en diseño de circuitos por computadora para aplicaciones espaciales, investigador en los Laboratorios Bell, así como profesor de la Universidad Case Western Reserve y Newark College of Engi neering, en los Estados Unidos. Fue el presidentefundador de la Academia Nacional de Ingeniería de México; vicepresidente y presidente del Consejo de Academias de Ingeniería y Ciencias Tecnológicas (organización mundial con sede en Washington que agrupa las Academias Nacionales de Ingeniería) y secretario de la Academia Mexicana de Ciencias. Actualmente es jefe de la Unidad de Enseñanza Auxiliada por Computadora de la División de Estudios de Posgrado de la Facultad de Ingeniería de la UNAM, investigador nacional en ingeniería, consejero educativo del MIT y consultor de la UNESCO. 